\title{
BMJ Open Can a nurse-led community-based model of hypertension care improve hypertension control in Ghana? Results from the ComHIP cohort study
}

\author{
Alma J Adler, ${ }^{1,2}$ Amos Laar, ${ }^{3}$ David Prieto-Merino, ${ }^{1}$ Reina M M Der, ${ }^{4}$ \\ Debbie Mangortey, ${ }^{4}$ Rebecca Dirks, ${ }^{5}$ Peter Lamptey, ${ }^{1}$ Pablo Perel ${ }^{1}$
}

To cite: Adler AJ, Laar A, Prieto-Merino D, et al. Can a nurse-led community-based model of hypertension care improve hypertension control in Ghana? Results from the ComHIP cohort study. BMJ Open 2019;9:e026799. doi:10.1136/ bmjopen-2018-026799

\section{- Prepublication history and} additional material for this paper are available online. To view these files, please visit the journal online (http://dx.doi. org/10.1136/bmjopen-2018026799).

AJA and $\mathrm{AL}$ contributed equally.

Received 2 October 2018 Revised 6 February 2019 Accepted 11 February 2019

Check for updates

(C) Author(s) (or their employer(s)) 2019. Re-use permitted under CC BY-NC. No commercial re-use. See rights and permissions. Published by BMJ.

For numbered affiliations see end of article.

Correspondence to

Dr Alma J Adler;

Alma.Adler@|shtm.ac.uk

\section{ABSTRACT}

Objectives To evaluate the effectiveness of the Community-based Hypertension Improvement Project (ComHIP) in increasing hypertension control.

Setting Lower Manya Krobo, Eastern Region, Ghana. Participants All adult hypertensive community members, except pregnant women, were eligible for inclusion in the study. We enrolled 1339 participants, $69 \%$ of whom were female. A total of 552 had a 6 -month visit, and 338 had a 12-month visit. Interventions We report on a package of interventions where community-based cardiovascular disease (CVD) nurses were trained by FHI 360. CVD nurses confirmed diagnoses of known hypertensives and newly screened individuals. Participants were treated according to the clinical guidelines established through the project's Technical Steering Committee. Patients received three types of reminder and adherence messages. We used CommCare, a cloud-based system, as a case management and referral tool.

Primary outcome Hypertension control defined as blood pressure (BP) under 140/90 mm Hg. Secondary outcomes: changes in BP and knowledge of risk factors for hypertension.

Results After 1 year of intervention, $72 \%$ (95\% Cl: $67 \%$ to $77 \%$ ) of participants had their hypertension under control. Systolic BP was reduced by $12.2 \mathrm{~mm}$ $\mathrm{Hg}(95 \% \mathrm{Cl}: 14.4$ to 10.1$)$ and diastolic BP by $7.5 \mathrm{~mm}$ $\mathrm{Hg}(95 \% \mathrm{Cl}: 9.9$ to 6.1). Due to low retention, we were unable to look at knowledge of risk factors. Factors associated with remaining in the programme for 12 months included education, older age, hypertension under control at enrolment and enrolment date. The majority of patients who remained in the programme were on treatment, with two-thirds taking at least two medications.

Conclusions Patients retained in ComHIP had increased BP control. However, high loss to follow-up limits potential public health impact of these types of programmes. To minimise the impact of externalities, programmes should include standard procedures and backup systems to maximise the possibility that patients stay in the programme.

\section{Strengths and limitations of this study}

- The community-based Hypertension Improvement Project is a large cohort study testing a community-based model of hypertension care.

- Trained community-based cardiovascular nurses conducted screening, diagnosis and management of hypertension patients.

- Patients were sent three types of short message service (SMS), daily reminders to take their medications, appointment reminders and weekly health education messages.

- Protocol stated that blood pressure would be checked with a minimum of three serial readings at regular intervals, but at a minimum of 6-monthly intervals.

A limitation of the study was that it did not include a control group.

\section{INTRODUCTION}

Globally, raised systolic blood pressure (SBP) is one of the greatest risk factors for disability. ${ }^{1}$ Hypertension is generally considered to be the level of raised $\mathrm{BP}$ where medications show a reduction in clinical events in randomised trials. This is generally accepted as $\geq 140$ SBP $\mathrm{mm} \mathrm{Hg}$ or $\geq 90$ diastolic $\mathrm{mm} \mathrm{Hg}$ (DBP). ${ }^{2}$

Evidence shows that lowering hypertensive individual's BP with antihypertensive drugs reduces the risk of further cardiovascular events; with a reduction in stroke by an estimated $35 \%-40 \%$ and a $20-25 \%$ reduction in myocardial infarction and heart failure. $^{3-5}$ While average age-standardised $\mathrm{BP}$ is decreasing in most high-income countries, it is increasing in most low-income and middle-income countries ${ }^{6}$ with $32 \%-50 \%$ of adults estimated to be hypertensive in sub-Saharan Africa. ${ }^{7}$

The Prospective Urban Rural Epidemiology study showed that despite high levels of hypertension worldwide, only $34 \%$ of 
Africans are aware of their hypertension status, only $31.3 \%$ receive any treatment and only $6.5 \%$ have their BP under control. ${ }^{8}$ Our recent study of hypertension prevalence in the Lower Manya Krobo, Ghana, showed that only $2.1 \%$ of hypertensives had their BP under control. ${ }^{9}$

Because of the great burden of hypertension in sub-Saharan Africa and the poor rate of hypertension control, innovative methods for hypertension management are needed. Launched in 2015, the Community-based Hypertension Improvement Project (ComHIP) introduced as an innovative model for hypertension control at the community level. ComHIP is a public-private partnership between the Ghana Health Service (GHS), FHI 360 and the Novartis Foundation.

The aim of ComHIP is to improve hypertension management and control in the Lower Manya Krobo District in the Eastern Region of Ghana. The programme includes a package of interventions composed of six components (online supplementary figure 1), aimed at increasing access to hypertension services at the community level. Screening in the community is provided by cardiovascular disease (CVD) nurses and community health officers (CHOs), as well as through local private sector drug shops called licensed chemical sellers (LCS). Ongoing hypertension management is provided by CVD nurses or, for those with comorbidities or severe conditions, at district hospitals. Patients are encouraged to routinely monitor their BP by having their BP measured at an LCS. The various service providers are linked through a cloud-based system, which revolves around bringing hypertension care into the community. Physicians, community-based CVD nurses, CHOs and LCS staff were trained by FHI 360 to provide specific services.

For instance, CVD nurses conduct hypertension screening, and confirmation of hypertension diagnosis, staging of the degree of hypertension, assessment of other CVD risk factors, counselling, monitoring and follow-up, and trained LCS conduct community BP screening and awareness raising. Further information can be found in the online supplementary material.

The ComHIP programme is being independently evaluated by the University of Ghana School of Public Health and the London School of Hygiene \& Tropical Medicine with a mixed-methods approach through a series of quantitative and qualitative studies. These studies include repeat cross-sectional surveys within the intervention and comparison districts to track overall awareness and prevalence of hypertension; a cohort of hypertensive persons included in ComHIP to assess hypertension control; a cost-effectiveness evaluation; a study to assess the level of patient-centeredness within the programme and a qualitative assessment of ComHIP stakeholders. In this paper, we report the results of the cohort study.

\section{Objectives}

The objective of this study was to evaluate the effectiveness of ComHIP for controlling hypertension in patients with hypertension enrolled in the ComHIP programme.

\section{METHODS}

\section{Study design}

The study was a prospective cohort study, which included all patients recruited into the ComHIP programme.

\section{Setting}

The study was conducted in Lower Manya Krobo, a municipality in the Eastern Region of Ghana. This is a periurban setting approximately 2 hours from the national capital, Accra, with a population of approximately 89246 , of whom $84 \%$ live in urban areas. ${ }^{10}$ Recruitment began in October 2015 and ended in December 2016.

\section{Training}

FHI 360 and the Ministry of health $(\mathrm{MoH})$ conducted training. Training duration ranged from 3 days for LCS and physicians, to 6 days for CVD nurses. Aside from the general training package (BP screening including the recommended standard operating procedures for BP checking, lifestyle modification counselling, interviewing/counselling techniques and treatment adherence counselling) offered to all personnel, CVD nurses and physicians received additional training on hypertension diagnosis, assessing the risk of patients, assessing for target organ damages (TODs), and training on drugs for the management of hypertension and their side effects and contraindications.

Participants were issued a certificate of participation signed by the cardiologist specialist who conducted the training and the director general of the GHS. Also, as is done by the GHS, the continuous learning logbooks of the GHS personnel were endorsed by the project to document the training received.

\section{Participants}

Patients were enrolled in the programme if (1) they were known hypertensives or (2) had an elevated BP reading at any ComHIP screening. Any individual living in Lower Manya Krobo, 18 years or older, was eligible, except pregnant women. Patients had to have access to a mobile phone to be enrolled in the programme. However, in order to negate the loss of patients, patients without phones were not necessarily excluded based on this; rather, they were encouraged to provide phone numbers of a willing third party who lived nearby.

\section{Intervention}

Community members were screened by CHOs, LCS or CVD nurses, using Omron M6 BP monitors that came with a cuff size of $42 \mathrm{~cm}$, the second largest cuff size in the market for those machines. Though the project requested for nurses to report cases of patient with bigger upper arms that required bigger cuff sizes, throughout the implementation, no such reports were received. The average of three serial readings was used to confirm hypertension diagnosis. Patients who were at risk of hypertension $(\mathrm{SBP} \geq 120$, but $<140$ ) were given health education. All patients with $\mathrm{SBP} \geq 140$ or $\mathrm{DBP} \geq 90$ were referred to a CVD nurse for diagnosis. Patients with $\mathrm{SBP} \geq 180$ or 
DBP $\geq 110$ were enrolled and referred to the physician for urgent care. Patients that were considered to have severe hypertension ( $\mathrm{SBP} \geq 180$ or $\mathrm{DBP} \geq 110$ or $\mathrm{SBP}$ between 160 and 179 or DBP 100 and 109 with one or more risk factors, or any evidence of organ damage see online supplementary appendix A) were referred for management by a physician at one of the district hospitals, until their BP was stable, and then they were returned to CVD nurses for care. All other patients were managed by CVD nurses.

Patients with hypertension were enrolled and followed for at least 1 year. All patients' interactions (with LCS, CHOs, CVD nurses, and community and hospital pharmacist and doctors) were recorded and uploaded through the CommCare platform. Patients were requested to present for appointments at the following intervals: monthly $\mathrm{BP}$ monitoring appointments; monthly, bi-monthly or quarterly review visits (depending on risk factors and personal factors) and 6-monthly follow-up assessments. Participants were recruited from October 2015 until December 2016, and followed through December 2017. Guidelines for patient visits can be found in the supplementary materials (online supplementary table 1, online supplementary figure 2 , online supplementary appendix A).

All enrolled participants were treated based on the same clinical guidelines established through the project's Technical Steering Committee, which included senior members of the GHS. The treatment goal was to improve BP of all patients to below 140/90 mm Hg. Participants were initiated onto drug therapy and supplemented with non-drug therapy (lifestyle modification including low-salt diets, increased fruit and vegetable diet, reduction in alcohol consumption, smoking cessation and regular aerobic exercise) irrespective of their risk level. The decision to initiate monotherapy or multiple drug therapy depended largely on the level at which the participant's BP was above goal and the overall risk level of patients. Recommended drugs and dosages are found in online supplementary table 2. Patient's response to antihypertensives was reviewed every 3 months if possible and modified based on recommended guidelines if required. In Ghana, there is a system of National Health Insurance (NHI) in which every Ghanaian is required to enrol. The scheme provides select medications at no cost for anyone who has a valid NHI card. Although the NHI scheme (NHIS) does not attempt to treat all diseases suffered by insured members, over $95 \%$ of disease conditions that afflict us are covered by the NHIS. Services can be accessed at accredited health facilities.

CommCare is a vital component of ComHIP. It serves as a case management system, referral tool and job aid for providers. The CommCare database is linked with an SMS platform to automatically send daily adherence reminders, weekly healthy living tips, and consultation and prescription refill reminders to enrolled patients. These messages are sent via text or voice SMS with four language choices. The programme is described in more detail elsewhere. ${ }^{9}$ Briefly, through CommCare, patients diagnosed with severe hypertension or coexisting conditions are automatically referred to a physician. All patients enrolled in ComHIP receive SMS daily for medication reminders, weekly for health education and appointment and screening reminders. CommCare also provides a cloud-based health records system that links patients' records with the SMS system. The SMS component of the project was implemented by a third party Viamo; to facilitate the link between the two systems, a bridge was built to automatically relay relevant information from the projects cloud-based health records to the Viamo messaging platform. To ensure confidentiality, only information relevant to schedule appointments is relayed to the Viamo platform (ie, patient code, date of visit, type of visit, next review or refill appointment, patient's phone number, preferred language, time of receipt of message and format of the message, referral details and BP). When a visit is missed, the system automatically relays back to the CVD nurse who enrolled the patient or is managing the patient via text message for the nurse to trace the patient. Due to operational problems, there was a break in service in CommCare that began on 12 May 2016 for a period of at least 3 months.

\section{Variables}

Main outcomes

The main outcomes of interest were hypertension control $(<140 / 90 \mathrm{~mm} \mathrm{Hg})$ and changes in SBP and DBP. Because of the low follow-up rate, we also used appointment around 6 months, and appointment around 12 months as outcomes of interest.

\section{Other variables}

Other variables included knowledge of risk factors for hypertension, demographic factors including age, gender and marital status, risk factors, such as body mass index (BMI), awareness of hypertension (defined as having knowledge of a previous diagnosis of hypertension), having hypertension under control prior to enrolment and having previous diagnoses of other heart diseases, and socioeconomic factors. A full list of variables is found in table 1.

\section{Data collection}

Data were collected on BP using standardised protocols. At 6 and 12 months, forms were administered by healthcare providers to collect information on patient knowledge of risk factors for hypertension and health behaviours.

All data were collected and downloaded from the CommCare platform. Initially, data were intended to be analysed from the patient knowledge/behaviour forms used at 6-month and 12-month follow-up appointments. Due to poor levels of follow-up, any appointment between 5 and 7 months after enrolment was used for the 6-month appointment analysis, and any appointment between 11 and 13 months after enrolment was used for the 12-month appointment analysis. 


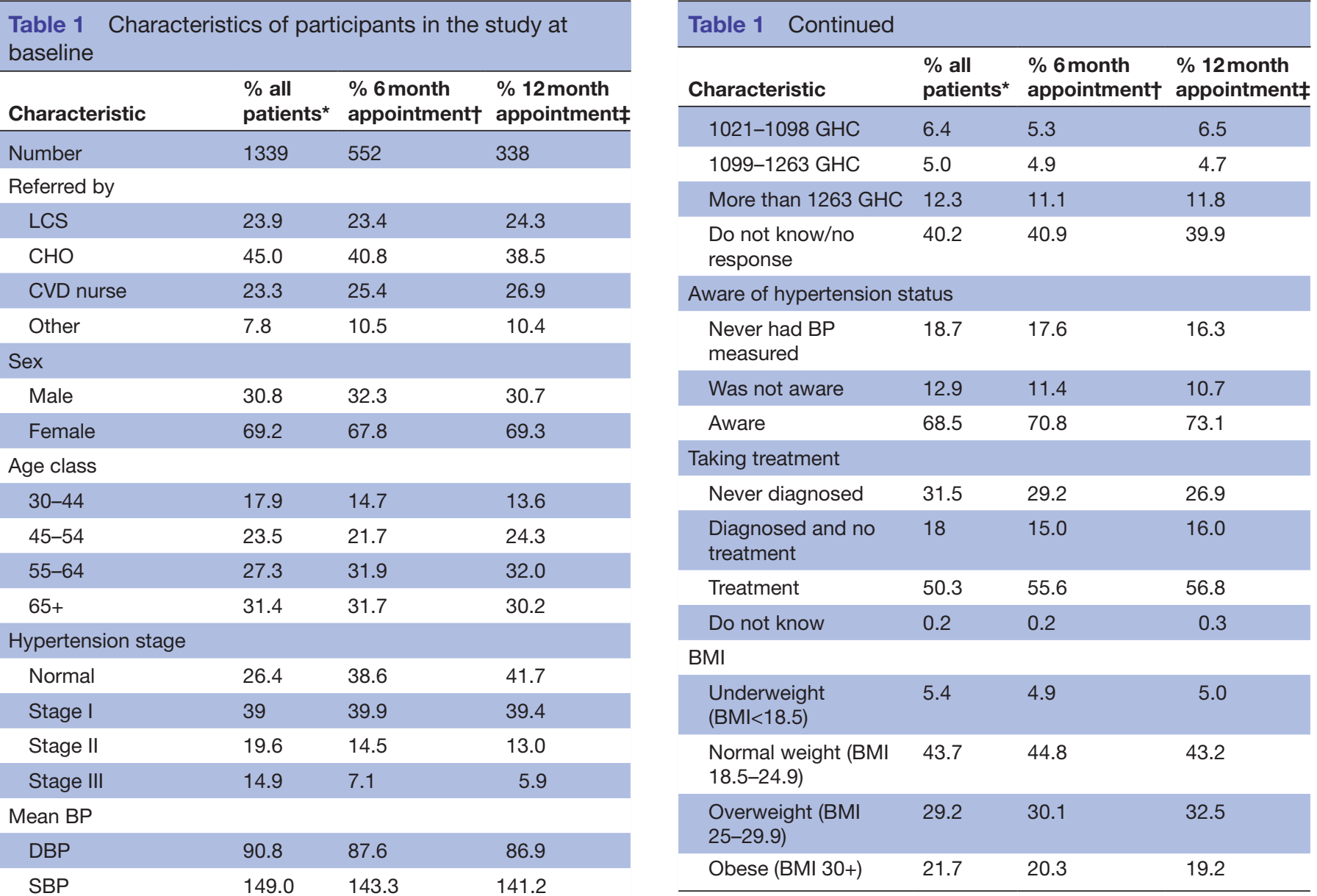

\begin{tabular}{|c|c|c|c|}
\hline \multicolumn{4}{|l|}{ Education } \\
\hline No formal education & 37.0 & 32.3 & 31.4 \\
\hline Primary & 41.7 & 431 & 45.6 \\
\hline Secondary & 16.1 & 18.5 & 14.8 \\
\hline Higher & 5.2 & 6.2 & 8.3 \\
\hline \multicolumn{4}{|l|}{ Ethnicity } \\
\hline Akan & 4.2 & 28.6 & 21.4 \\
\hline Dangme & 69.5 & 42.2 & 26 \\
\hline Ewe & 22 & 39.3 & 22.4 \\
\hline Other or do not know & 4.3 & & \\
\hline \multicolumn{4}{|l|}{ Religion } \\
\hline Christian & 96 & 97.6 & 97.9 \\
\hline Muslim & 3.2 & 1.5 & 1.2 \\
\hline Traditional & 0.5 & 0.4 & 0.3 \\
\hline None & 0.3 & 0.5 & 0.6 \\
\hline \multicolumn{4}{|l|}{ Marital status } \\
\hline Never married & 5.7 & 5.1 & 3.6 \\
\hline Married/cohabiting & 54.4 & 54.7 & 57.7 \\
\hline Separated/divorced & 5.5 & 14.1 & 15.4 \\
\hline Widowed & 26.1 & 25.9 & 23.1 \\
\hline No response & 0.2 & 0.2 & 0.3 \\
\hline \multicolumn{4}{|l|}{ Household income } \\
\hline Less than $728 \mathrm{GHC}$ & 18.7 & 17.0 & 17.2 \\
\hline 728-1020 GHC & 17.4 & 20.8 & 19.8 \\
\hline
\end{tabular}

${ }^{*}$ All patients with hypertension enrolled in the cohort.

†Patients with hypertension with 6-month appointment/follow-up. łPatients with hypertension with a 12-month appointment/followup.

$\mathrm{BMI}$, body mass index; $\mathrm{BP}$, blood pressure; $\mathrm{CHO}$, community health officers; CVD, cardiovascular disease; DBP, diastolic blood pressure; LCS, licensed chemical sellers; SBP, systolic blood pressure.

\section{Sample size}

This cohort study included all the patients recruited in the ComHIP programme and a specific sample size was not calculated. However, in the protocol, we assumed that the total district population is about 90000 ; about 30000 of whom are adults, and about 36\% (12 000) are estimated to be hypertensive. Assuming that about $10 \%$ of the adults with hypertension in the district will be included in the ComHIP programme, we would have a cohort of 1200 patients with hypertension.

We estimated that a cohort study of 1200 patients with hypertension would provide a power greater than $90 \%$ (with an alpha error of 0.05) to detect a twofold increase of control of hypertension (from $4 \%$ to $8 \%$ ).

\section{Patient and public involvement}

Community members, including community leaders, were first involved through a stakeholder workshop. In this workshop, community members shared their thoughts, knowledge and concerns about health in 
general, Non-communicable disease-related conditions and access to healthcare. Furthermore, community members were made aware of the hypertension project planned to be initiated in their community. This information was considered in finalising the design of the service delivery model and the development of prevention, education and behaviour change messages.

Patients were recruited into the project through free screening offered at (1) local drug shops, names LCS; (2) Community Health Planning Service (CHPS) sites or (3) community pharmacies. There were community screening activities and radio programmes through which community members were educated on the project and hypertension in general. In addition, ComHIP staff conducted annual stakeholder meetings to provide updates to community members on the project progress.

\section{Statistical methods}

We recoded exposures to reduce the number of levels and for missing values, for all the previous diagnosis/ awareness, we have coded 'missing' or 'not known' or 'no answer' as 0 , so that value 1 always means 'patient knows of a previous diagnosis' while value 0 means anything else (patient does not know or answer is missing). Because there were few previous diagnoses of each specific event (MI, stroke, diabetes etc), we created a variable with value 1 if any diagnosis was present and 0 if none was present.

For education, we assumed that those that did not know (48) or did not respond (26) did not have previous formal education (the largest group). We then grouped education in four levels: (1) no formal education, (2) primary (completed or not), (3) secondary (completed or not) and (4) higher (university).

For marital status, we made four categories: (1) never married, (2) married or cohabiting, (3) separated or divorced and (4) widowed.

We described the distribution of each variable at baseline, 6 months and 12 months follow-up, although comparisons cannot be done directly due to the large number of individuals that did not have a follow-up. To study what variables might affect the patient staying for 12 months in the programme, we ran a logistic regression for the binary outcome variable: "patient had 12-month visit $(\mathrm{Y} / \mathrm{N})$ '. To consider the loss to follow-up (patterns of visits), we separated the individuals into four different groups: (A) those individuals that did not come to any follow-up visit, (B) those that came only to the 6-month visit, (C) those that came only to the 12-month visit and (D) those that came to both follow-up visits.

We described the absolute values of BP (SBP and DBP), the proportion of patients with $\mathrm{BP}$ under control and the distribution of hypertension stages for each of these groups in each of the visits. We estimated the average changes of BP for each group at each follow-up visit and we compared the changes between groups with Student's t-tests. We compared the mean of SBP and DBP between the groups with analysis of variance (ANOVA) models. To compare the proportion of patients with hypertension control or the distribution of hypertension stages between groups, we used $\chi^{2}$ tests. To test the changes of variables within groups, we used paired t-tests for continuous variables and marginal homogeneity tests for categorical variables.

\section{RESULTS}

\section{Participants}

A total of 18339 individuals, 18 years and older, were screened, 4118 were referred to CVD nurses to confirm diagnosis, and of those 1339 were enrolled, 76 (5.7\%) were considered low-risk (stage 1BP, which is SBP 140-159 or DBP 90-99 without any TODs, comorbidities or $\geq 2$ risk factors), $559(41.7 \%)$ were moderate risk (stage 2 , which is SBP 160-179 or DBP 100-109 without any TODs, comorbidities or $\geq 2$ risk factors or stage $1 \mathrm{BP}$ with TODs, comorbidities or $\geq 2$ risk factors) and 704 (52.6\%) were considered highrisk (stage 3, which is $\mathrm{SBP} \geq 180$ or $\mathrm{DBP} \geq 101$ without any TODs, comorbidities or $\geq 2$ risk factors or stage $2 \mathrm{BP}$ with TODs, comorbidities or $\geq 2$ risk factors).

\section{General characteristics of the cohort}

The average age of the cohort was 58 years. Everyone was enrolled into the cohort by CVD nurses. Of the 1339 people enrolled in the cohort, $24 \%$ were referred to ComHIP by LCS, $45 \%$ were referred by CHO, $23 \%$ were referred by CVD nurses, 3\% were through physicians and 5\% were referred through other channels. About $69 \%$ of the cohort was female and $31 \%$ male. Other characteristics of people enrolled in the cohort are found in table 1.

\section{Other risk factors}

About $5.4 \%$ of the sample was underweight, $43.7 \%$ was normal, $29.2 \%$ was overweight and $21.7 \%$ was obese. The mean BMI at enrolment in the cohort was 26.1 (95\% CI: 25.82 to 26.4 ).

We did not analyse smoking, cholesterol or diabetes as only $1 \%$ of the sample were smokers, $3.5 \%$ reported having had a previous cholesterol test and only $28 \%$ had a previous diabetes test.

\section{$\mathrm{BP}$ at enrolment}

The average SBP was 147.2 (SD 22.1) $\mathrm{mm} \mathrm{Hg}$, and average DBP was 89.9 (SD 13.3) mm Hg. At enrolment, 917 (68.5\%) had a previous diagnosis of hypertension, of which 654 $(71.3 \%)$ were already taking some antihypertensives, and $297(32.4 \%)$ had their BP under control.

\section{BP management}

Of 1339 enrolled in the study, 712 (53.2\%) did not come for a follow-up (group A), 289 (21.6\%) had only a 6-month visit (group B), 75 (5.6\%) had only the 12-month visit (group C) and $263(19.6 \%)$ had both visits (group D). In total, only 552 $(41 \%)$ had a 6 -month follow-up appointment, and only 338 (25\%) had a 12-month follow-up appointment.

Loss to follow-up and characteristics of those who stayed in the study

Patients with their hypertension already under control were more likely to present for care. The variable that showed the 
Table 2 Multivariate analysis of baseline characteristics associated with staying in the programme for 12 months

\begin{tabular}{|c|c|c|}
\hline & OR $(95 \% \mathrm{Cl})$ & $P$ value \\
\hline Enrolled year prior to the break & $0.46(0.35$ to 0.60$)$ & 0.00 \\
\hline Sex & $0.88(0.63$ to 1.24$)$ & 0.48 \\
\hline Age (1-year increments) & 1.01 (1.00 to 1.02$)$ & 0.03 \\
\hline BMI & 1.00 (0.97 to 1.02$)$ & 0.90 \\
\hline \multicolumn{3}{|c|}{ Education reference category: no formal education } \\
\hline Primary education & 1.41 (1.03 to 1.93$)$ & 0.03 \\
\hline Secondary education & $1.13(0.73$ to 1.73$)$ & 0.59 \\
\hline Higher education & 2.42 (1.33 to 4.43$)$ & 0.004 \\
\hline \multicolumn{3}{|l|}{$\begin{array}{l}\text { Reference category: never } \\
\text { married }\end{array}$} \\
\hline Married/cohabitating & 1.77 (0.90 to 3.48$)$ & 0.10 \\
\hline Separated/divorced & $1.86(0.90$ to 3.87$)$ & 0.10 \\
\hline Widowed & 1.27 (0.61 to 2.64$)$ & 0.52 \\
\hline Household size & 1.01 (0.95 to 1.07$)$ & 0.69 \\
\hline Hypertension control & 1.93 (1.47 to 2.54$)$ & $<0.001$ \\
\hline Awareness of hypertension & $1.00(1.00$ to 1.00$)$ & 0.97 \\
\hline Hypertension treatment & 1.00 (0.99 to 1.00$)$ & 0.33 \\
\hline Any other previous diagnosis & 0.86 (0.69 to 1.07$)$ & 0.18 \\
\hline $\begin{array}{l}\text { Confidence in management } \\
\text { of hypertension }\end{array}$ & $1.00(1.00$ to 1.01$)$ & 0.63 \\
\hline
\end{tabular}

BMI, body mass index.

greatest association with likelihood of having a 6-month or 12-month appointment was enrolment month. Participants who enrolled earlier were much more likely to stay in the programme than those who enrolled later (table 1).

Multivariate analysis suggested that recruitment before 12 May 2016 (1year before the break in service), age, education and hypertension under control in the first visit showed significant associations with having a 12-month appointment. Recruitment after 12 May 2016 reduced the chances of coming to further visits, the older the patient and the higher the education level, the higher the chances that the patient would come to the follow-up visits. Patients with controlled hypertension at enrolment were nearly twice as likely to come to follow-up visits. None of the other variables showed significant associations (table 2).

\section{Changes in $\mathrm{BP}$}

Because 12-month follow-up was below 30\%, we did not look at overall changes in BP, but we did look at overall changes in $\mathrm{BP}$ in those that remained in the study at 6 and 12 months.

On average, patients who enrolled and presented for a follow-up appointment at around 6months had a $10.3 \mathrm{~mm} \mathrm{Hg}$ reduction in SBP (95\% CI: 12.0 to 8.6 ) and a $6.3 \mathrm{~mm} \mathrm{Hg}$ reduction in DBP (95\% CI: 7.2 to 5.2 ) (table 3). There was a greater reduction in those patients who had a follow-up appointment at 1year, when there was a $12.2 \mathrm{~mm} \mathrm{Hg}$ reduction (95\% CI: 14.4 to 10.1 ) in SBP and a $7.5 \mathrm{~mm} \mathrm{Hg}$ (95\% CI: 9.9 to 6.1$)$ reduction in DBP after 1 year in the programme. Not all patients who had a 12-month appointment also had a 6-month appointment, $263 \mathrm{had}$ both, and they had an $11.9 \mathrm{~mm} \mathrm{Hg}$ reduction (95\% CI: -14.3 to -9.5 ) in SBP and $7.1 \mathrm{~mm} \mathrm{Hg}$ reduction (95\% CI: -8.6 to -5.5 ) in DBP (table 3 ).

\section{Knowledge of risk factors}

Because of the poor retention, we were unable to comment on knowledge or risk factors.

There was also a significant reduction in hypertension stage, with a lower percentage of patients with hypertension having stage III hypertension over time (table 4).

\begin{tabular}{|c|c|c|c|c|c|c|c|}
\hline \multirow[b]{2}{*}{ Groups } & \multirow[b]{2}{*}{$\mathbf{N}$} & \multirow[b]{2}{*}{ Visit } & \multicolumn{2}{|l|}{ SBP } & \multicolumn{2}{|l|}{ DBP } & \multirow{2}{*}{$\begin{array}{l}\text { HT control } \\
\%(95 \% \mathrm{Cl})\end{array}$} \\
\hline & & & Mean (SE) & $\begin{array}{l}\text { Mean difference } \\
(95 \% \mathrm{Cl})\end{array}$ & Mean (SE) & $\begin{array}{l}\text { Mean difference } \\
(95 \% \mathrm{Cl})\end{array}$ & \\
\hline \multirow[t]{3}{*}{ All patients } & 1339 & E & $147.2(0.60)$ & & $89.9(0.36)$ & & $31 \%$ (29\% to $34 \%)$ \\
\hline & 552 & $6 \mathrm{~m}$ & $132.9(0.80)$ & $-10.3(-12.0$ to -8.6$)$ & $81.3(0.47)$ & $-6.3(-7.3$ to -5.2$)$ & $69 \%$ (65\% to $73 \%)$ \\
\hline & 338 & $12 m$ & $128.9(1.05)$ & $-12.2(-14.4$ to -10.1$)$ & $79.4(0.61)$ & $-7.5(-8.9$ to -6.1$)$ & $72 \%$ (67\% to $77 \%)$ \\
\hline (A) No visits & 712 & $\mathrm{E}$ & $150.4(0.85)$ & & $91.7(0.49)$ & & $25 \%$ (21\% to $28 \%)$ \\
\hline \multirow[t]{2}{*}{ (B) Only $6 \mathrm{~m}$} & 289 & $E$ & $146.4(1.28)$ & & $89.0(0.77)$ & & $34 \%$ (29\% to $40 \%)$ \\
\hline & & $6 \mathrm{~m}$ & $135.7(1.15)$ & $-10.1(-13.2$ to -8.1$)$ & $82.7(0.68)$ & $-6.3(-7.8$ to -4.8$)$ & $61 \%(55 \% \text { to } 67 \%)^{*}$ \\
\hline \multirow[t]{2}{*}{ (C) Only $12 \mathrm{~m}$} & 75 & $\mathrm{E}$ & $145.9(2.62)$ & & $90.2(1.63)$ & & $36 \%$ (25\% to $48 \%$ ) \\
\hline & & $12 m$ & $132.5(2.56)$ & $-13.5(-18.5$ to -8.6$)$ & $81.0(1.38)$ & $-9.2(-12.4$ to -6.0$)$ & $71 \%(59 \% \text { to } 81 \%)^{*}$ \\
\hline \multirow[t]{3}{*}{ (D) 6 and $12 \mathrm{~m}$} & 263 & E & $139.8(1.18)$ & & $86.1(0.80)$ & & $43 \%$ (37\% to $50 \%)$ \\
\hline & & $6 \mathrm{~m}$ & $129.8(1.08)$ & $-10.0(-12.2$ to -7.7$)$ & $79.8(0.63)$ & $-6.3(-7.8$ to -4.8$)$ & $77 \%(72 \% \text { to } 82 \%)^{*}$ \\
\hline & & $12 \mathrm{~m}$ & $127.9(1.13)$ & $-11.9(-14.3$ to -9.5$)$ & $79.0(0.67)$ & $-7.1(-8.6$ to -5.5$)$ & $72 \%(66 \% \text { to } 78 \%)^{*}$ \\
\hline
\end{tabular}

${ }^{*}$ The comparison of these intervals with enrolment visit of the same group produces all $p$ values $<0.0001$.

BP, blood pressure; DBP, diastolic blood pressure; E, enrolment; HT, hypertension, m, month; SBP, systolic blood pressure; SE, standard error 
Table 4 Distribution of hypertension stage in each group in each visit. The $p$ values are extracted from: (1) $X^{2}$ tests to compare that row with group A of no follow-up and (2) from marginal homogeneity tests comparing the distribution of the same group in enrolment visit

\begin{tabular}{|c|c|c|c|c|c|c|c|}
\hline Groups by patterns of visits & $\mathbf{N}$ & Visit & No HT & Stage I & Stage II & Stage III & $P$ value \\
\hline \multirow[t]{2}{*}{ All patients } & 1339 & $1 \mathrm{~m}$ & $31.0 \%$ & $39.0 \%$ & $18.4 \%$ & $11.6 \%$ & \\
\hline & 552 & $6 \mathrm{~m}$ & $68.7 \%$ & $19.7 \%$ & $9.4 \%$ & $2.2 \%$ & $<0.001(2)$ \\
\hline (A) No follow-up & 712 & $1 \mathrm{~m}$ & $24.6 \%$ & $38.7 \%$ & $21.5 \%$ & $15.2 \%$ & \\
\hline (B) Only 6-m visit & 289 & $1 \mathrm{~m}$ & $34.3 \%$ & $39.4 \%$ & $17.0 \%$ & $9.3 \%$ & $0.002(1)$ \\
\hline \multirow[t]{2}{*}{ (C) Only 12-m visit } & 75 & $1 \mathrm{~m}$ & $36.0 \%$ & $36.0 \%$ & $17.3 \%$ & $10.7 \%$ & $0.167(1)$ \\
\hline & 75 & $12 \mathrm{~m}$ & $70.7 \%$ & $14.7 \%$ & $10.7 \%$ & $4.0 \%$ & $<0.001(2)$ \\
\hline \multirow[t]{3}{*}{ (D) 6-m and 12-m visit } & 263 & $1 \mathrm{~m}$ & $43.4 \%$ & $40.3 \%$ & $11.8 \%$ & $4.5 \%$ & $<0.001(1)$ \\
\hline & 263 & $6 \mathrm{~m}$ & $77.2 \%$ & $14.8 \%$ & $6.5 \%$ & $2.2 \%$ & $<0.001(2)$ \\
\hline & 263 & $12 \mathrm{~m}$ & $72.3 \%$ & $20.9 \%$ & $5.3 \%$ & $1.5 \%$ & $<0.001(2)$ \\
\hline
\end{tabular}

m, month.

\section{Awareness}

Overall awareness of hypertension status in the overall cohort was $68.5 \%$ at enrolment. Individuals who stayed in the programme longer were more likely to be aware of their hypertension status. About $70.8 \%$ of individuals who stayed in the programme for 6 months were aware of their hypertension status, and $73.1 \%$ of those who stayed in the programme for 12 months were aware of their hypertension status (table 1 ).

Treatment

Treatment increased between enrolment and 6-month and 12-month appointments. Although only $44.2 \%$ of patients were receiving any medication at enrolment, the majority were being treated at 6 months $(90.4 \%)$ and at 12 months $(92.2 \%)$. At enrolment, the majority of patients who were on treatment were taking a calcium channel blocker (CCB) (36\% of all patients), but at 6 months, the majority were on diuretics $(75.9 \%)$ followed by a CCB (69.5\%). The same pattern was found at 12 months with $79.8 \%$ taking diuretics, and $71.5 \%$ taking a CCB (table 5 ).

In patients who had a 6-month appointment, $24.1 \%$ were taking only one medication, $32 \%$ were taking two medications and over $30 \%$ were taking more than two medications. In patients who had a 12-month appointment, $23 \%$ were taking one medication, $32.6 \%$ were taking two medications and over $32 \%$ were taking more than two medications.

\section{Control}

There was an increase in BP control in patients who remained in the programme (table 3); however, patients who stayed in the programme were more likely to have

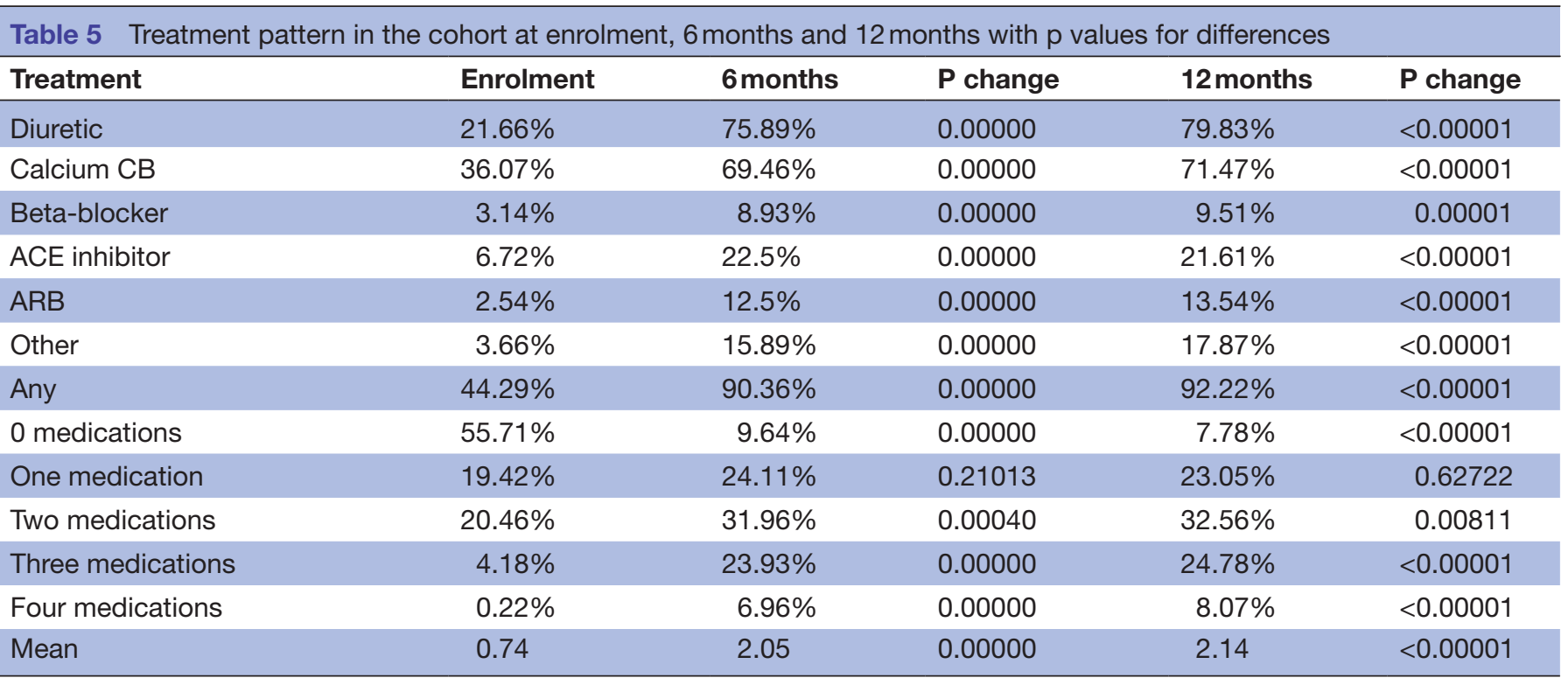

$\mathrm{ACE}$, angiotensin converting enzyme; $\mathrm{ARB}$, angiotensin receptor blockers; $\mathrm{CB}$, channel blocker. 
their BP under control on enrolment. In the group of patients that did not have a second appointment (group A), the baseline BP control was $25 \%$ while in the other groups (B, C and D) was $34 \%, 36 \%$ and $43 \%$, respectively. These differences were statistically significant (table 4 ). The BP control increased to $69 \%$ (95\% CI: $65 \%$ to $73 \%$ ) in the individuals that visited at 6 months. In the patients that had the 12-month visit, the control increased to $72 \%$ (95\% CI: $67 \%$ to $77 \%$ ). Of patients who had both a 6-month and 12-month follow-up appointment, the control increased to $77 \%$ (95\% CI: $72 \%$ to $82 \%$ ) at 6 months, but slightly decreased to $72 \%$ at 12 months (95\% CI: $66 \%$ to $78 \%$ ) (table 3 , table 4 ).

\section{DISCUSSION}

\section{Summary of results}

Of 1339 patients enrolled in ComHIP, only 552 (41\%) had a follow-up appointment at 6 months, 338 (25.2\%) had a follow-up appointment at 12months and 263 (20\%) had both 6-month and 12-month appointments. Participants who had more education, were older, had their hypertension under control at enrolment, or who had the opportunity to spend at least a year in the programme before the break in service were more likely to attend appointments at 6 and/or 12 months.

Among the group of patients who continued in the programme for 6 or 12 months, we found strong evidence of a reduction in DBP and SBP, and an increase (from under half to more than two-thirds) of hypertension control. We also found strong evidence of an increase of the patients under treatment, of the number of medications received per patient and a decrease in the number of individuals with severe hypertension.

\section{Comparison with other studies}

Other studies evaluating task sharing for hypertension management have shown modest levels of success. For example, one randomised controlled study conducted in Ghana using task sharing (but supplying free medications) showed greater reductions in SBP in patients randomised to the arm that included trained nurses, as compared with the one that just provided free medications and health insurance. ${ }^{11}$

The poor follow-up reported in our study is not unexpected. Many studies have shown poor levels of follow-up or adherence to clinic appointments. In one study conducted in three primary care clinics in Kibera, Kenya, between 2010 and 2012, 1465 hypertensive or diabetic patients were identified. Of these, $31 \%$ of patients were lost to follow-up. Of these, $55 \%$ of non-diabetic patients had their BP under control by 24 months, but only $28 \%$ of diabetic patients. ${ }^{12}$

In another study conducted in Kibera, Kenya, between 2015 and 2016, 3861 patients with hypertension were identified in health centres or clinics. Of those, 3069 patients did not complete 6 months of follow-up (79\%).
Of those, patients who remained in the programme over 6 months, they found $63 \%$ adherence to appointments. ${ }^{13}$

In a study conducted in the slums of Nairobi, only $3.4 \%$ of participants showed completed compliance with the programme. About $30 \%$ only showed up for one appointment, and 5\% only had two visits. Similar to our study, they found that patients who remained in the programme showed significant reductions in SBP and DBP. ${ }^{14}$

In a study done in two sites (one rural and one urban) in Malawi, of 4075 patients referred for clinical care, only $61 \%$ attended their referral appointments. Of those, $47 \%$ of patients with hypertension were still in contact after 24 months. Similar to our findings, they found uptake in care to be higher in older patients, being on antihypertensives prior to enrolment, and not being in employment. Unlike our study, they found that females were more likely to be retained in care. ${ }^{15}$

Similarly, a study of hypertensive and diabetic patients in rural Cameroon found that only $18.1 \%$ of participants were still in care after 1 year. However, similar to our study, they found significant decreases in SBP and DBP in patients with hypertension with at least two documented visits.

\section{Strengths and limitations}

A major strength of this study is unlike most other hypertension programmes, ComHIP uses existing GHS protocols and medications and does not require outside funds or intervention for medications. This means that there is a much greater chance of long-term sustainability of the programme as it does not rely on outside sources for medications.

Limitations of the study include that data were only available for encounters with service providers within the ComHIP network. Any appointments with doctors and pharmacists (licensed or unlicensed) that were not part of ComHIP would not have been registered, so it is possible that patients were obtaining antihypertensives from non-licensed sellers, which would not be captured in the ComHIP database. Another limitation of ComHIP was that the cohort did not have a control.

Due to the extremely poor follow-up, it is not possible to generalise our findings regarding the impact on $\mathrm{BP}$ control to other studies, other than to emphasise the importance of effective strategies to promote follow-up. Finally, it is important to remember that nearly $70 \%$ of the initial cohort was aware of their hypertension status and about half were taking medications, which is a much higher proportion than in the general population. While this was done in ComHIP to ensure access to hypertension management to community members who otherwise would not have been able to access services, it is an important consideration when considering generalisability to the overall population.

\section{Interpretation}

In $25 \%$ of people who had a 12-month appointment, there was strong evidence of an increase of the patients 
receiving medications, the average number of medications received per patient and the level of hypertension control; we also found a reduction in both BP and hypertension status. However, like most other studies in the region, the high loss to follow-up highlights that innovative hypertension programmes, such as ComHIP, need to develop better ways to retain patients within the programme.

Community-based hypertension programmes in resource-poor setting often are complex to carry out and are prone to poor follow-up. There are many possible reasons that follow-up in our study was low.

The factor most associated with retention in the programme was enrolment date. This is significant as due to operational issues, there was a gap of CommCare utilisation for 3 months. Anecdotally, FHI 360 ComHIP staff learnt that this gap in CommCare service had caused both service providers and staff to believe that the intervention had stopped, which may have resulted in a low rate of completion of follow-up appointments. Considering difficulties associated with community-based studies in low-resource settings, it is imperative to ensure continuity of service. Other factors that could cause this association may be healthcare professional fatigue; engaging patients to present for appointments may require considerable effort, such as multiple phone calls and personal interaction, for which the CVD nurses did not receive additional monetary compensation. It is possible that over time, the enthusiasm of the CVD nurses for the intervention may have waned. Also, as in any low-resource settings, there is a great deal of workforce turnover; FHI 360 recognised this early in the implementation and trained extra staff to bridge the gaps; however, it is still possible that new healthcare providers who replaced them may not have had the same level of training. A complementary component of the evaluation that includes qualitative research with different ComHIP stakeholders is underway to analyse in depth the possible reasons that may have caused people to not adhere to the programme. (See Adler et al Barriers and facilitators to the implementation of a community-based hypertension improvement project in Ghana: a qualitative study and Laar et al Health system challenges to hypertension and related non-communicable diseases prevention and treatment: perspectives from Ghanaian stakeholders.)

Lastly, our study found that older individuals were more likely to continue in care, this was found in at least one other study ${ }^{15}$ but was not reported on in most studies. This could be because older patients may have more time to attend clinics. Patients with their hypertension under control were about twice as likely to stay in the programme. This is not surprising as they had already exhibited better health-seeking behaviours.

\section{Recommendations}

For patients enrolled and who continued in the programme, we found an important impact on the management of hypertension and in BP control.
However, the high loss to follow-up of patients recruited limits the potential public health impact of these types of programmes. In order to minimise the impact of externalities (such as the CommCare service gap in ComHIP), programmes should have standard procedures and backup systems to maximise the possibility that patients stay in the programme, particularly younger and less educated individuals. Also, appropriate incentives should be put in place to keep programme staff fully engaged and avoid programme fatigue. Future studies should further identify causes of loss to follow-up and find effective ways to adapt programmes accordingly (eg, access to treatment within the community and targeted behaviour change messaging) to ensure that most of the patients recruited stay long-term in the programme. Future research may also want to focus on more difficult to reach patients who have lower levels of awareness and treatment on enrolment.

\section{Author affiliations}

${ }^{1}$ Department of Non-communicable Disease Epidemiology, London School of Hygiene \& Tropical Medicine, London, UK

${ }^{2}$ Department of Global Health and Social Medicine, Harvard Medical School, Boston, Massachusetts, USA

${ }^{3}$ Department of Population, Family \& Reproductive Health, School of Public Health, University of Ghana, Accra, Ghana

${ }^{4} \mathrm{FHI} 360$, Accra, Ghana

${ }^{5} \mathrm{FH} 360$, Washington, DC, USA

Acknowledgements We are grateful to all of the cohort clients. We are also grateful to the leadership and political support of the Ghana Health Service (both from the national and district levels) for their support.

Contributors PL conceived the idea of the project, and PL and RD designed the interventions. PL, AA, AL and PP designed the research component of the project. $\mathrm{DM}$ and RMMD supervised the implementation of the programme. RMMD is the programme data manager. AA and DP-M performed the statistical analyses. AA drafted the manuscript, with inputs from all the authors. All authors read and approved the final version of the manuscript.

Funding Funds for the project were made available by Novartis Foundation, Basel, Switzerland.

Disclaimer Novartis Foundation did not have any input or control over this manuscript.

Competing interests Co-authors PL, AA, AL, PP, and DP-M worked on the ComHIP Programme for which their institutions (LSHTM and UGSPH) have received grants from the Novartis Foundation. Co-authors RD, RMMD and DM are staff of the FHI 360, which provided technical direction to ComHIP implementation.

Patient consent for publication Not required.

Ethics approval Ethical approval was granted by the Institutional Review Boards (IRBs) of LSHTM (LSHTM Ethics Ref: 10152), the Ghana Health Service (ID No. GHS-ERC 04/01/15) and the University of Ghana at Noguchi Memorial Institute for Medical Research (Ethics clearance \# IRB00001276).

Provenance and peer review Not commissioned; externally peer reviewed.

Data sharing statement Summary statistics related to the data set used in the project are available by request.

Open access This is an open access article distributed in accordance with the Creative Commons Attribution Non Commercial (CC BY-NC 4.0) license, which permits others to distribute, remix, adapt, build upon this work non-commercially, and license their derivative works on different terms, provided the original work is properly cited, appropriate credit is given, any changes made indicated, and the use is non-commercial. See: http:// creativecommons.org/licenses/by-nc/4.0/. 


\section{REFERENCES}

1. GBD 2016 DALYs and HALE Collaborators. Global, regional, and national disability-adjusted life-years (DALYs) for 333 diseases and injuries and healthy life expectancy (HALE) for 195 countries and territories, 1990-2016: a systematic analysis for the Global Burden of Disease Study 2016. Lancet 2017;390:1260-344.

2. WHO. 1999 World Health Organization-International Society of Hypertension Guidelines for the Management of Hypertension. Guidelines Subcommittee. J Hypertens 1999;17:151-83.

3. Ying A, Arima H, Czernichow S, et al. Blood Pressure Lowering Treatment Trialists' Collaboration. Effects of blood pressure lowering on cardiovascular risk according to baseline body-mass index: a meta-analysis of randomised trials. Lancet 2015;385:867-74.

4. Collins R, Peto R, Godwin J, et al. Blood pressure and coronary heart disease. Lancet 1990;336:370-1.

5. Law MR, Morris JK, Wald NJ. Use of blood pressure lowering drugs in the prevention of cardiovascular disease: meta-analysis of 147 randomised trials in the context of expectations from prospective epidemiological studies. BMJ 2009;338:b1665.

6. Olsen MH, Angell SY, Asma S, et al. A call to action and a lifecourse strategy to address the global burden of raised blood pressure on current and future generations: the Lancet Commission on hypertension. Lancet 2016;388:2665-712.

7. Addo J, Smeeth L, Leon DA. Hypertension in sub-saharan Africa: a systematic review. Hypertension 2007;50:1012-8.

8. Chow CK, Teo KK, Rangarajan S, et al. Prevalence, awareness, treatment, and control of hypertension in rural and urban communities in high-, middle-, and low-income countries. JAMA 2013;310:959-68.
9. Lamptey P, Laar A, Adler AJ, et al. Evaluation of a community-based hypertension improvement program (ComHIP) in Ghana: data from a baseline survey. BMC Public Health 2017;17:368.

10. GSS. Population by Region, District, Locality of Residence, Age Groups and Sex, 2010. Accra, Ghana: Ghana Statistical Service. 2012 http://www.statsghana.gov.gh/docfiles/population_by_region_ district_locality_of_residence_age_groups_and_sex,_2010.pdf (Accessed 22 Nov 2016).

11. Ogedegbe G, Plange-Rhule J, Gyamfi J, et al. Health insurance coverage with or without a nurse-led task shifting strategy for hypertension control: A pragmatic cluster randomized trial in Ghana. PLoS Med 2018;15:e1002561.

12. Sobry A, Kizito W, Van den Bergh R, et al. Caseload, management and treatment outcomes of patients with hypertension and/or diabetes mellitus in a primary health care programme in an informal setting. Trop Med Int Health 2014;19:47-57.

13. Kuria N, Reid A, Owiti P, et al. Compliance with follow-up and adherence to medication in hypertensive patients in an urban informal settlement in Kenya: comparison of three models of care. Trop Med Int Health 2018;23:785-94.

14. Werner ME, van de Vijver S, Adhiambo M, et al. Results of a hypertension and diabetes treatment program in the slums of Nairobi: a retrospective cohort study. BMC Health Serv Res 2015; $15: 512$.

15. Musicha C, Crampin AC, Kayuni N, et al. Accessing clinical services and retention in care following screening for hypertension and diabetes among Malawian adults: an urban/rural comparison. $J$ Hypertens 2016;34:2172-9. 